\title{
Mémoire, histoire et patrimoine
}

\author{
Une illustration : la pratique de \\ l'histoire vivante médiévale
}

\author{
Audrey Tuaillon Demésy \\ (Docteur en sociologie, Université de Franche-Comté)
}

\section{Résumé/Abstract}

[Fr] L'histoire vivante est une pratique sociale et culturelle, souvent de loisir, parfois professionnelle, qui consiste à mettre en vie une manière de faire d'un temps passé. Elle permet d'exposer et de donner à penser le concept de mémoire. Celui-ci doit être analysé en relation avec les notions d'histoire et de patrimoine. Le travail de terrain mené autour de ces espaces de sociabilité est basé sur des méthodes empruntées à l'ethnologie et à la sociologie (observations participantes, entretiens, enquête quantitative). La mémoire personnelle, mobilisée par les enquêtés à travers le champ sémantique de l'ancestralité, est associée à la dimension historique de la démarche mise en place par le groupe, qui se reconnaît dans une forme de passé en commun. Par ailleurs, le rapport au patrimoine favorise le passage d'une mémoire «figée », morte à une autre, vivante et réactualisée, symbole de la reconstitution historique. Mots-Clés : histoire, mémoire, Moyen Âge, musée, patrimoine, reconstitution.

[En] Living history is a cultural or social (recreational or professional) practice which allows for an explanation and an examination of the concept of memory. The latter must be understood as related to history and heritage. The completed fieldwork borrows from ethnological and sociological methods (participant observation, qualitative and quantitative research.) Autobiographical memory displayed by the community (through the semantic field of ancestrality) is linked to the historical dimension of the approach set up by the group, which turns to a shared memory of the past. Furthermore, the link to its heritage allows the group to move from a dead, «Fixed memory to a living, reactualised, symbol of historical reconstitution.

Keywords: Heritage, History, Memory, Middle Age, Museum, Reenactment.

\section{Introduction}

L'histoire vivante est une pratique sociale et culturelle, souvent de loisir, parfois professionnelle, qui consiste à mettre en vie une manière de faire d'un temps passé. Ses objectifs visent à exposer de façon interactive des fragments d'événements ou d'activités historiques. Elle se décline selon plusieurs époques, de la préhistoire à la se- 
conde guerre mondiale en passant par l'Antiquité, le Moyen Âge ou la Révolution. Elle comprend deux facettes, distinctes mais complémentaires, que sont la reconstitution historique et les Arts martiaux historiques européens, couramment appelés AMHE. Ceux-ci visent à présenter, à l'aide de sources primaires, des techniques martiales d'affrontement normé ; ils fonctionnent comme une activité physique et se pratiquent avec du matériel contemporain (masque d'escrime, armes neutralisées, etc.). Quant à la reconstitution, elle se pratique en costume et cherche à représenter une personne et, plus précisément, un statut social, conforme à la période historique choisie.

Dans le cadre de la recherche menée ici, la période concernée de l'histoire vivante est le Moyen Âge. C'est à partir d'un travail de terrain ethnographique, réalisé en France, en Suisse et en Belgique, complété par des données recueillies à l'aide d'entretiens et de questionnaires, que l'analyse s'est mise en place. La participation à des évènements de reconstitution ou d'AMHE a permis de saisir le phénomène étudié d'un point de vue « interne ». Il s'agissait aussi bien de rassemblements réservés aux initiés ayant pour objectifs premiers les échanges et l'apprentissage de l'artisanat, que de manifestations publiques visant à exposer aux visiteurs une autre manière d'appréhender l'histoire et le passé, en dehors des livres et des supports «figés». L'histoire vivante comprend des caractéristiques multiples, mobilisant les concepts de transmission, d'identité, de lien social ou encore de loisirs et de professionnalisation. Toutefois, c'est la notion de «mémoire » qui sera ici exposée, notamment dans son lien avec l'histoire : comment est-il possible de passer d'une filiation personnelle, reliée à la tradition, à une mémoire collective ou partagée ? De même, le patrimoine, qu'il soit bâti ou institué dans les musées, favorise l'activation mémorielle, en tant que support d'expression d'un passé à sauvegarder. Ce qui est ici en jeu est l'articulation possible de façon conjointe de plusieurs concepts qui avoisinent souvent celui de mémoire, dans le cadre spécifique de l'activité étudiée : l'histoire vivante médiévale en tant que démarche culturelle.

\section{D'une mémoire historique à la formation d'une mémoire partagée}

En premier lieu, le lien entre mémoire et histoire peut être abordé de deux façons distinctes. Les acteurs de l'histoire vivante évoquent fréquemment la notion d'«ancêtres » à laquelle ils rattachent leur pratique. Pourtant, le passé réellement pris en compte n'en demeure pas moins immatériel, en ce qu'il se révèle déshumanisé.

Les enquêtés utilisent peu le vocable de mémoire et lui préfèrent d'autres mots : « histoire », «ancêtres », « autrefois », «avant », etc. Pourtant, en plus d'une poursuite historique, les pratiquants de l'histoire vivante médiévale cherchent à inscrire leur démarche dans une continuité mémorielle, pour lui donner corps et la rattacher à 
du « vivant ». Les deux conceptions d'histoire et de mémoire sont exposées de manière conjointe : la première renvoie au support de connaissances, alors que la seconde permet un rapprochement entre « autrefois » et « aujourd'hui ». Par ailleurs, le discours recueilli sur le terrain permet de faire le lien entre ce qui relève de $l^{\prime}$ « historique » et de ce qui relève de la «mémoire». Faire référence à des «ancêtres » renvoie en effet à une conception humanisée de l'époque médiévale. Ces individus-référents dans les propos tenus peuvent être personnels, «mes [ancêtres] », ou communs à plusieurs personnes : «nos ». De même, ils peuvent être pluriels («prédécesseurs »), ou indéfinis («on »). Les termes employés restent souvent vagues. Lorsque l'approche est globale, «nos ancêtres» par exemple, le pronom possessif renvoie à un collectif qui englobe sans nul doute la communauté des pratiquants, mais certainement aussi un ensemble plus vaste, constitué par le public potentiel, ramené à l'ensemble des Français ou même des Européens. Cette impossibilité à situer précisément les référents se comprend en tenant compte de la distance temporelle qui les sépare de ceux qui en parlent. Pourtant, la référence est bien présente. La notion d'ancestralité renvoie à une inscription dans un passé indéterminé, le Moyen Âge n'étant même pratiquement jamais évoqué à titre de précision.

Ce qui est mis en avant est une filiation technique, artisanale entre les « ancêtres » médiévaux et l'activité contemporaine ; il n'est d'ailleurs presque jamais fait mention d'une volonté de retrouver un état d'esprit. En outre, ce qui est avancé est une impossibilité d'échapper au conditionnement contemporain. Autrement dit, les pratiquants s'inscrivent pleinement dans la société moderne et avancent le fait que de nombreux changements culturels ont amené des transformations dans les mentalités. Dès lors, ils acceptent les limites de leur démarche et ne cherchent pas à retrouver des manières de penser qui seraient «médiévales » : les référents (sociaux, culturels, politiques, économiques, etc.) ayant changé, il apparaît comme impossible de «se mettre à la place » de personnes ayant vécu au Moyen Âge. Pour cette raison, la relation entre l'historique et la mémoire porte uniquement sur le rapport au corps. C'est bien ce dernier qui fait la liaison entre un passé ancestral et la démarche actuelle. La «trace » permet l'inscription mémorielle.

«Évoquer les traces, c'est se référer à ce qui subsiste d'un passé [...]. Elles ont toujours intéressé les hommes dans la mesure où elles matérialisent ce qui a disparu, lui donnent une image, permettent de se le représenter, de l'étudier, de se souvenir, de commémorer, de montrer une évolution en remontant le temps. » (Boursier $2001: 1$ )

Dans le cadre de mon objet d'étude, la trace mobilisée est le geste, et plus largement tout ce qui permet de reconstituer un artisanat médiéval. Tout se passe comme si le corps était une permanence humaine susceptible de donner à voir un geste historique. Il en est de même pour les AMHE : «retrouver» le geste martial dépend bien du corps mis en scène, qui doit se mettre en adéquation avec des techniques passées. Mobiliser une mémoire corporelle autorise les initiés à mettre du sens sur leur savoir-faire, à les ancrer dans une mémoire qui se justifie par un nécessaire be- 
soin de replacer un artefact dans son contexte de production. Cependant, bien que la mobilisation d'une mémoire corporelle fasse sens pour les enquêtés, elle n'est pas nécessairement révélatrice ni garante d'une historicité avérée, mais elle permet de situer les savoir-faire dans un ancrage mémoriel, nécessaire à la pratique. C'est par exemple le cas pour les reproductions de bijoux ou de techniques du «feutre » qui vont parfois emprunter des éléments à d'autres périodes historiques ou à d'autres espaces géographiques. Ainsi, certains artisans évoquent les façons de faire du XIX siècle comme modèle réapproprié dans leur démarche, en insistant sur la permanence historique des méthodes (ré)utilisées.

La «source », en tant que terme fréquemment mentionné pour justifier d'une reconstitution ou d'un attachement particulier à un savoir, permet de faire le lien entre les connaissances, la mémoire et l'histoire. La filiation mémorielle est, dans ce cas précis, une filiation historique, qui se réalise à travers une gestuelle corporelle précisément définie. Ce double vocabulaire employé peut s'analyser au regard de ce que démontre Pierre Nora : «La mémoire sourd d'un groupe qu'elle soude, ce qui revient à dire, comme Halbwachs l'a fait, qu'il y a autant de mémoires que de groupes ; qu'elle est, par nature, multiple et démultipliée, collective, plurielle et individualisée. L'histoire, au contraire, appartient à tous et à personne, ce qui lui donne vocation à l'universel. » (Nora 1997 : 25) Ce double discours permet de conserver une cohérence et une vision unifiée des raisons qui poussent à la pratique de l'histoire vivante, et qui la motivent. Le désir de rattacher l'histoire à la mémoire permet de rendre la première plus proche et de lui donner un sens autrement inaccessible. Pourtant, la précision ne va jamais au-delà, afin de ne pas entrer en contradiction avec une réalité qui présente une filiation impossible à prouver au niveau personnel. L'utilisation par les enquêtés de la notion d'ancestralité cristallise dans les discours le rapport entretenu à la mémoire; l'enjeu est de pouvoir mettre du réel et du concret, ou plus simplement de la vie, sur des traces qui demeurent autrement inertes.

Si le lien entre mémoire et histoire se comprend du point de vue des enquêtés, la véritable relation qui existe entre les personnes physiques médiévales et les acteurs contemporains est invérifiable. C'est pourquoi cette inscription dans une filiation, bien que nécessaire, apparaît comme illusoire. Pourtant, l'idée d'une continuité temporelle est unanimement partagée par les enquêtés, même si elle n'est pas toujours énoncée. L'idée est de donner à voir une pratique méconnue mais qui pourtant s'inscrit dans une continuité historique. La mémoire est un socle favorisant une transmission indispensable, pour un besoin de reconnaissance des activités, mais aussi des connaissances et savoir-faire appris. Mais de quelle mémoire s'agit-il exactement ?

Celle-ci ne peut être personnelle, aucun enquêté n'ayant pu dire qu'il avait un ascendant reconnu au Moyen Âge, mais tous s'accordent sur une temporalité et un lieu commun en référence à la pratique, et plus largement, sur le concept de tradition : «tradition martiale », « arts traditionnels », « gestes traditionnels », etc. La tradition renvoie bien ici à une filiation ascendante. La «mémoire » peut être à son tour mo- 
bilisée pour l'éclairer. Spontanément, le vocable de tradition est davantage employé par les usagers que celui de mémoire, sans doute parce qu'il est moins polysémique et permet, une fois encore, de ne pas situer dans le temps ce sur quoi porte la tradition, évitant ainsi le conflit entre histoire et mémoire. Pourtant, ce consensus à propos de reconstitutions traditionnelles invite à interroger la notion de mémoire collective.

En premier lieu, lorsque les reconstituteurs, ou les pratiquants d'AMHE, font appel à une mémoire, celle-ci doit être précisée. Halbwachs en distingue ici deux différentes :

«Durant le cours de ma vie, le groupe national dont je faisais partie a été le théâtre d'un certain nombre d'événements [...]. Je porte avec moi un bagage de souvenirs historiques, que je peux augmenter par la conversation ou par la lecture. Mais c'est là une mémoire empruntée et qui n'est pas la mienne [...]. Il y aurait donc lieu de distinguer en effet deux mémoires, qu'on appellerait, si l'on veut, l'une intérieure ou interne, l'autre extérieure, ou l'une mémoire personnelle, l'autre mémoire sociale. Nous dirions plus exactement encore: mémoire autobiographique et mémoire historique. » (Halbwachs 1968 [1950] : 36-37)

Dans cette optique, la référence faite par les enquêtés aux «ancêtres » ne peut faire sens pour une mémoire autobiographique et ce qui éclaire leur discours est alors une mémoire historique, dans le sens que lui donne Halbwachs, en ce qu'elle s'appuie sur des traces spécifiques (archives, enluminures, etc.) qui vont permettre une forme de codification et d'unification mémorielle. Une superposition est ainsi faite par les pratiquants de l'histoire vivante médiévale entre mémoire historique et mémoire personnelle. Cet amalgame, comme on l'a vu, permet de maintenir une cohésion dans l'activité et de lui donner vie, tout en fournissant une identité de groupe aux enquêtés. L'aspect immatériel et déshumanisé (suite au manque de filiation directe), est « oublié » au profit d'une mémoire partagée, qui trouve ses racines dans le principe de transmission et de perpétuation de gestes techniques.

Il s'agit alors d'aborder la notion de mémoire collective en tant que support permettant l'appartenance à un groupe particulier et qui cherche justement à préserver une mémoire historique. «La mémoire collective est traitée dans sa menace même de disparition. Une quantité singulière de traces peut être préservée d'autant plus que les derniers représentants de l'époque se passionnent pour la reconstitution de leur propre vie sociale et culturelle. » (Jeudy 1986 : 26) Pour ce qui est plus précisément de l'histoire vivante, il convient certainement davantage de parler de « mémoire partagée » plutôt que de «mémoire collective ». La reconstitution, comme les AMHE, n'existent réellement en France que depuis une quinzaine d'années ; pour cette raison, la reconnaissance sociale de ces deux pratiques n'est pas encore complètement opérée et les adhérents demeurent, à l'heure actuelle, peu nombreux au regard d'autres activités de loisirs. Ainsi, plutôt que de parler de mémoire collective, il semble préférable d'employer le terme de mémoire partagée, entendue à l'échelle du 
groupe des pratiquants. Il n'en demeure pas moins que cette forme de mémoire s'imbrique avec une autre, historique.

À cet égard, la question des «traces » prend une place essentielle pour les enquêtés, qui s'appuient sur les données fournies par l'archéologie ou l'histoire pour leur démarche. D'un point de vue effectif, les informateurs ne sont pas les «derniers représentants de l'époque », puisqu'ils n'ont pas de lien direct avec le Moyen Âge, mais leur discours les place comme un groupe social particulier, pour qui la sauvegarde d'une vision spécifique de l'époque médiévale, à savoir l'importance des techniques et de la lutte contre les idées préconçues sur cette période, forme un enjeu commun. À ce titre, ils se positionnent comme les garants d'une mémoire des savoir-faire en perdition, souvent peu ou non prise en compte par les institutions culturelles, qui accordent davantage d'importance à l'histoire "nationale », comprise au sens de «Grande Histoire ». Le renvoi à une mémoire partagée instaure alors une identité propre au groupe des pratiquants.

La production d'une mémoire reconnue et acceptée par les enquêtés repose sur les fondements d'une mémoire historique, enrichie par les traces laissées par le passé. Les cadres sociaux qui fondent une mémoire particulière correspondent à l'identité du groupe des acteurs de l'histoire vivante, représentée par leur volonté commune : la mise en place de gestes techniques, la diffusion de connaissances, l'envie de se rattacher à un passé qui fait sens pour eux. Halbwachs rappelle d'ailleurs que «reproduire n'est pas retrouver : c'est, bien plutôt, reconstruire» (Halbwachs 1976 [1925] : 92). Une distinction doit être opérée entre une mémoire historique figée et une mémoire partagée par le groupe. Il s'agit bien d'inscrire la pratique dans le cadre d'une « reconstruction », plutôt que dans celle d'une « remémoration », qui apparaît comme impossible. D'ailleurs, la pratique actuelle de l'histoire vivante ne symbolise pas la recherche d'un passé jugé plus attrayant: les enquêtés ne rejettent pas le monde moderne et ne cherchent pas refuge dans une époque révolue, mais revendiquent au contraire une démarche basée sur la transmission et l'immersion dans un temps «autre », durant un week-end, à la manière de vacances hors de chez soi. En effet, les différentes manifestations favorisent les échanges et, par extension, la création d'une mémoire commune, qui donne une identité au groupe. La circulation des connaissances et savoir-faire techniques se réalise autant à l'intérieur de la communauté des pratiquants qu'en dehors. Au cours de manifestations off, c'est-à-dire fermées au public, les reconstituteurs peuvent échanger sous la forme d'ateliers: les connaissances sont transmises sous l'angle de l'expérimentation et de la démonstration. Il peut s'agir d'apprendre à faire du naalbinding (technique de tressage aux doigts), de tourner de la céramique ou encore de tester une «coulée de bronze». D'un autre côté, lors d'événements ouverts au public, la transmission est avant tout destinée aux visiteurs. L'enjeu est d'apporter aux non-pratiquants des connaissances davantage théoriques (le public ne participe pas aux ateliers) et de présenter une vision du Moyen Âge en dehors des clichés habituels. Ainsi, le matériel militaire est 
exposé (souvent soupesé par le public), afin de rappeler, par exemple, qu'une épée ne pèse pas lourd, que les chevaliers en armure - représentation par excellence de la période médiévale - sont apparus très tardivement compte tenu du fait que le Moyen Âge dure mille ans, etc. L'objectif est également de montrer que cette époque est bien différente de l'image que peuvent en donner les films ou mêmes les livres d'histoire: un combat ne durait que quelques secondes et la période n'est pas aussi «sombre» que le laisse croire l'imagination collective. Il convient également de préciser que la mémoire mobilisée l'est dans un cadre permettant les échanges : en aucun cas les pratiquants ne sont «nostalgiques » d'une époque passée. Ils ne rejettent pas le monde contemporain, mais cherchent au contraire à se servir des traces laissées par l'histoire pour comprendre certains aspects de la vie médiévale. Ainsi, la mémoire historique (des connaissances acquises relatives à l'époque médiévale) se transforme peu à peu en mémoire partagée, propre au groupe, qui met en avant les principes d'une continuité des gestes techniques et de leur nécessaire transmission à la postérité. Ce changement va permettre une forme d'assignation identitaire qui détermine les frontières du groupe des pratiquants.

Un point doit aussi être fait en ce qui concerne la mémoire « institutionnelle », qui peut être suscitée dans plusieurs cadres. L'histoire vivante se situe à la croisée de plusieurs institutions : les archives lui servent de support pour ses investigations ; la recherche est un socle commun au groupe, qui permet de lui conférer une mémoire partagée et, par extension, une identité ; enfin, l'enseignement, ou tout du moins la transmission d'une mémoire ainsi mobilisée, est l'un des vecteurs principaux par lesquels se donne à voir la pratique. Mais une opposition peut se ressentir entre une mémoire privée et une mémoire institutionnelle, qui ne correspondent pas toujours. Ce qui doit être relevé est le décalage entre une mémoire instituée, celle des livres par exemple, et une mémoire sinon privée, du moins partagée par le groupe des pratiquants. Un décalage est bien perceptible, en ce que les seconds ne reconnaissent pas la vision qu'ils cherchent à présenter du Moyen Âge dans la mémoire instituée, reconnue et acceptée par tous. Un rapport de force risque d'être établi, d'autant plus que les ouvrages scolaires ne répondent pas toujours aux attentes des enquêtés. Cependant, l'utilisation de l'histoire vivante à titre de complément par les écoles ou les musées, permet de défaire une tension éventuelle. La place de chacune des mémoires présentées reste définie et elles ne viennent généralement pas en opposition frontale et directe.

La constitution d'une mémoire «partagée », sinon collective, permet, à travers les différents cadres sociaux au sein desquels elle est mobilisée (regroupements entre participants, transmission, langage employé par les enquêtés dans lequel les autres se reconnaissent, etc.), de modifier la mémoire historique de l'ensemble du groupe. Chaque manifestation apportant son lot de connaissances, la mémoire historique prend vie à travers une mémoire partagée. Les deux sont articulées, le seconde ayant besoin d'être entretenue au regard de la première. 


\section{Histoire vivante et sauvegarde patrimoniale}

Il s'agit à présent d'orienter la réflexion du côté du patrimoine et des lieux qui donnent à voir de la mémoire. Tout d'abord, la mémoire permet de sélectionner ce qui peut faire l'objet d'une patrimonialisation.

«Le patrimoine est le produit d'un travail de mémoire qui, au fil du temps et selon des critères très variables, sélectionne certains éléments hérités du passé pour les ranger dans la catégorie des objets patrimoniaux [...]. » (Candau 2005 : 119)

Pour l'objet d'étude, le patrimoine bâti, sous-entendu les châteaux, édifices religieux, etc., ne forme pas un lieu d'expression de l'histoire vivante. S'il ne fait pas sens pour les acteurs, c'est en partie car il n'est que rarement le reflet, le support mémoriel de l'époque médiévale, beaucoup de ces monuments ayant été reconstruits par la suite. Pour autant, d'autres bâtiments viennent s'inscrire dans une démarche mémorielle. Ces reconstitutions, initiées par des pratiquants, ne sont pas faites de pierres, mais de matériaux beaucoup plus «périssables » dans le temps. Ainsi en estil des maisons paysannes, durant quasiment toute la période médiévale. Il s'agit de reconstruire ce qui ne se donne pas à voir ailleurs et le bâtiment apparaît comme un socle de mémoire pertinent dans le cadre plus global de la démarche. Construire une ferme mérovingienne ou une bergerie $d u X^{\mathrm{e}}$ siècle entre bien dans ce processus : c'est toute une vision particulière du passé qui est en jeu. L'appui mémoriel qui donne à voir le Moyen Âge n'est plus simplement le « château-fort », mais aussi des maisons paysannes qui constituaient la grande majorité des habitations médiévales. La question du «monument» doit être traitée à l'aune de son enjeu de mémoire. Ce qui se joue est la mise en avant d'une vision différente de la période, en dehors des consensus habituellement admis. La mémoire de ce qui aurait pu être vu à cette époque est bien une motivation essentielle avec, pour corollaire, une exposition en dehors des cadres communément reconnus de la représentation des monuments médiévaux. Toutefois, les animations dans les châteaux, par exemple, ne sont pas rejetées et il faut préciser que l'ensemble des classes sociales spécifiques à l'époque médiévale sont représentées par la reconstitution. Pour autant, l'enjeu des enquêtés est de mettre en avant une forme d'éducation active et populaire : en ce sens, les lieux habituellement peu investis par le public sont privilégiés comme étant de nouveaux supports de transmission des connaissances.

D'autres institutions reliées au patrimoine renferment aussi des supports de mémoire : ce sont les musées. Il s'agit de passer de l'enjeu patrimonial des musées à leur enjeu mémoriel. En tant que soutiens d'une mémoire matérielle, les musées présentent des objets, véritables reflets d'une époque particulière. Ils sont le prolongement concret de la mémoire historique. Par ailleurs, leur objectif est la «conservation », le maintien en l'état un objet, tandis que l'histoire vivante se place du côté de la «sauvegarde», mettant en jeu d'autres notions, dont celle de mémoire partagée qui permet le changement, puisqu'elle est susceptible d'évoluer avec le groupe (de 
nouvelles façons de faire un artisanat peuvent apparaître, comme d'autres manières de transmettre aux visiteurs). La place de l'histoire vivante au sein des musées peut se comprendre au regard du rapport entretenu à la mémoire. Si les musées donnent à voir une mémoire «morte», inanimée et figée dans les vitrines, symbole d'une époque révolue, la reconstitution, mais tout autant les AMHE, présentent au contraire une mémoire vivante, volonté revendiquée de sauvegarde d'un patrimoine historique et technique. L'envie de présenter des savoir-faire «en action » (geste martial, démonstration de poterie ou de forge, etc.) qui utilisent parfois des objets reconstitués d'après les originaux présents dans les musées, renvoie bien à une sensibilité particulière, qui fait appel à une mémoire mettant en scène « du passé », appuyée et illustrée par les reconstitutions et mises en vie de gestes et de techniques historiquement possibles.

D'autre part, la mémoire permet de sélectionner ce qui peut faire l'objet d'une patrimonialisation. Mémoire et patrimoine semblent indissociablement liés, la première favorisant l'inscription de ce qui dit être conservé dans le domaine du second. Cette idée se retrouve pour l'histoire vivante. Les AMHE présentent ainsi un renouveau quant à l'approche martiale : la volonté de «retrouver» des techniques de combat s'appuie autant sur un phénomène mémoriel (notamment parce que leur ancrage est très ciblé : en Europe, par opposition aux arts martiaux orientaux), que sur un fait patrimonial. Les discours portent sur des «arts du combat traditionnels », renvoyant, une fois encore, à une filiation ou tout du moins, à une continuité temporelle. Cette idée se retrouve aussi dans le cadre de la reconstitution : ce qui motive les enquêtés est un souhait de réactualiser les «connaissances de nos origines et la conservation d'un savoir transmis ». Mémoire et patrimoine sont compris ensemble : il s'agit d'acquérir des compétences liées à un passé indéfini, de les conserver puis de les transmettre.

Un autre point qui explique la présence de la mémoire dans la démarche est la peur latente de l'effacement et, plus encore, de la mort. Pour pallier cela, à cet effroi de voir disparaître un patrimoine commun, la mémoire peut apparaître comme un remède. Le but est de « faire la preuve » (Fourcade 2007 : 526) de l'intérêt de sauvegarder les connaissances et les savoir-faire propres à la période médiévale. Assister à l'effacement du passé semble impossible pour les pratiquants, qui mettent en œuvre des stratégies de sauvegarde (à travers les prestations culturelles associatives, la réhabilitation de monuments, etc.). Il convient de passer d'une mort imminente d'une forme de culture historique (entendue au sens de la somme des connaissances et artefacts propres à une époque délimitée) à une mémoire «figée » dans les musées, jusqu'à parvenir à la mise en vie de cette même mémoire par les animations proposées. Le lien est ainsi fait avec une mémoire «vivante » qui tend à mettre en scène des activités pour lutter contre une mort annoncée par les vitrines des musées, figées dans le temps. 
Enfin, les actions effectuées à destination d'une sauvegarde du patrimoine peuvent se comprendre en lien avec une recherche de lien social. «Concrètement, ce qui accompagne les activités associatives autour du patrimoine conçu comme mémoire, c'est le processus d'interconnaissance que favorisent les concertations, les expositions ou les manifestions. » (Glevarec \& Saez 2002 : 273) Ici, la vie du groupe au cours des différents événements lui donne sens : il s'agit essentiellement des normes et valeurs communément admises et adoptées par les pratiquants (notamment : acceptation des limites de la démarche, soutien et entraide qui vont permettre une de cohésion sociale interne, partage des savoirs, etc.). Ces manières de faire autorisent à réajuster une certaine forme de mémoire partagée, en tant qu'échange de concepts et connaissances à propos de l'époque reconstituée. Autrement dit, un apport mémoriel commun cristallisé dans la dimension patrimoniale accorde une identité commune aux pratiquants, et encourage une perpétuation de la démarche d'histoire vivante par les liens ainsi créés entre les individus.

D'une mémoire morte à mémoire vivante, le lien entretenu au patrimoine permet de comprendre les enjeux spécifiques de la reconstitution, qui compose avec les supports mémoriels «figés », dans les musées ou les bâtiments, afin de leur redonner une place centrale dans ses activités.

\section{Conclusion}

« Conserver », « sauvegarder», «re-constituer », sont donc des termes essentiels et fréquemment utilisés par l'histoire vivante, qui cherche à construire une mémoire particulière, mettant en avant ce qui a été laissé de côté par ailleurs. Mais cette boulimie mémorielle dont elle fait preuve s'inscrit dans le cadre plus global de la société dans son ensemble. Nora rappelle qu' «aucune époque n'a été aussi volontairement productrice d'archives que la nôtre, non seulement par le volume que sécrète spontanément la société moderne, non seulement par les moyens techniques de reproduction et de conservation dont elle dispose, mais par la superstition et le respect de la trace » (Nora $1997:$ 31). De ce fait, l'histoire vivante englobe plusieurs manières de concevoir le rapport au passé : mémoire historique, mémoire partagée ou collective, mémoire vivante ou patrimoniale. Mais quelle que soit la façon dont elle est envisagée, la mémoire renvoie toujours à une identité construite par les enquêtés : celle d'appartenir à un groupe particulier, qui partage des valeurs communes et une vision similaire du Moyen Âge. En conclusion, l'histoire vivante présente une activité spécifique d'accès à une culture historique, en jouant avec les notions de «mémoire », d'« ancestralité » ou encore de «patrimoine ». Cette activité donne lieu à plusieurs manières d'aborder et de concevoir le passé mais, à chaque fois, les interactions entre les individus constituent la forme à travers laquelle se véhiculent les conceptions mémorielles en présence. 


\section{Bibliographie}

Andrieux J.-Y. (1997). Patrimoine et histoire. Paris: Belin.

Boursier J.-Y. (2001). «Le monument, la commémoration et l'écriture de l'Histoire ». Socio-anthropologie (9) [En ligne].

Candau J. (2005). Anthropologie de la mémoire. Paris: Armand Colin.

Ferro M. (2008). «Regard sur les guerres de mémoires dans le monde ». In P. Blanchard, M. Ferro et I. Veyrat-Masson (sous la dir. de). Les Guerres de mémoires dans le monde. Paris: CNRS Édition.

Fourcade M.-B. (2007). «La mise en ligne des mémoires du génocide arménien ». Ethnologie française vol. 37(3). 525-531.

Glevarec H., Saez G. (2002). Le Patrimoine saisi par les associations. Paris: La Documentation française.

Halbwachs M. (1968). La Mémoire collective. Paris: PUF (1 $1^{\text {re }}$ éd. : 1950).

Halbwachs M. (1976). Les Cadres sociaux de la mémoire. Paris: Archontes ( $1^{\mathrm{re}}$ éd. : 1925).

Jeudy H.-P. (1986). Mémoires du social. Paris: PUF.

Munier-Temime B. (dir.) (2007). Sur les voies du patrimoine. Entre culture et politique. Paris: L'Harmattan.

Nora P. (dir.) (1997). Les Lieux de mémoire (3 vol.). Paris: Gallimard.

Ricoeur P. (2000). La Mémoire, l'histoire, l'oubli. Paris: Seuil. 